\title{
Continuum of care approach for managing non-communicable diseases in low- and middle- income countries
}

\author{
Rajshree Thapa, Ayse Zengin, Amanda G Thrift
}

Department of Medicine, School of Clinical Sciences at Monash Health, Monash University, Melbourne, Australia

A continuum of care approach is an important public health tool that can provide crucial understanding into all stages of disease progression, from prevention through to long-term care, and would enable consolidation of efforts to control NCDs. Strategies must be strengthened to improve outcomes across the NCD continuum, from prevention through to long-term care.
1 The Lancet report on the non-communicable diseases (NCDs) countdown reveals that the current progress is sub-optimal. Indeed, at current rates of decline, 86 countries (46\%) for women and 97 countries (52\%) for men, will fail to achieve the sustainable development goal (SDG) target by 2030 [1]. High income countries (HICs) have been successful in reducing mortality from NCDs (cardiovascular disease, diabetes, cancer and chronic respiratory disease), although mainly from reductions in cardiovascular deaths. This decline in cardiovascular mortality has been achieved by a rapid progress in both prevention and treatment, including declines in cigarette smoking, improvements in controlling hypertension, and use of statins, thrombolysis and stents [2]. Unfortunately, cardiovascular deaths, which form the largest proportion of deaths from NCD, are not declining in most Sub-Saharan and Central Asian countries [3] and there are instances where such mortality has even increased in as many as 15 countries for women and 24 countries for men [1].

Collaborative efforts are required to curb the NCD burden and mortality in these countries. A continuum of care approach is an important public health tool that can provide crucial understanding into all stages of disease progression, from prevention through to long-term care, and would enable consolidation of efforts to control NCDs (Figure 1). While the model has been widely used in managing chronic conditions such as HIV [5], it has not been optimally used to monitor the impact across the various spectra of NCD care.

There is already compelling evidence for targeting services across the continuum of care for NCDs: prevention, diagnosis, treatment and long-term care [4]. Ford et al. reported that evidence-based medical and surgical treatments reduced cardiovascular deaths deaths in USA by $47 \%$, while reductions in major risk factors reduced cardiovascular deaths by 44\% [6]. Owolabi proposed a stroke quadrangle comprising primary and secondary prevention, access to well-equipped care and rehabilitation and research networks to address the burden of stroke in Africa [7]. Countries in Sub-Saharan Africa, Central and South Asia still face a challenge across the continuum as routine health check-ups and/or 


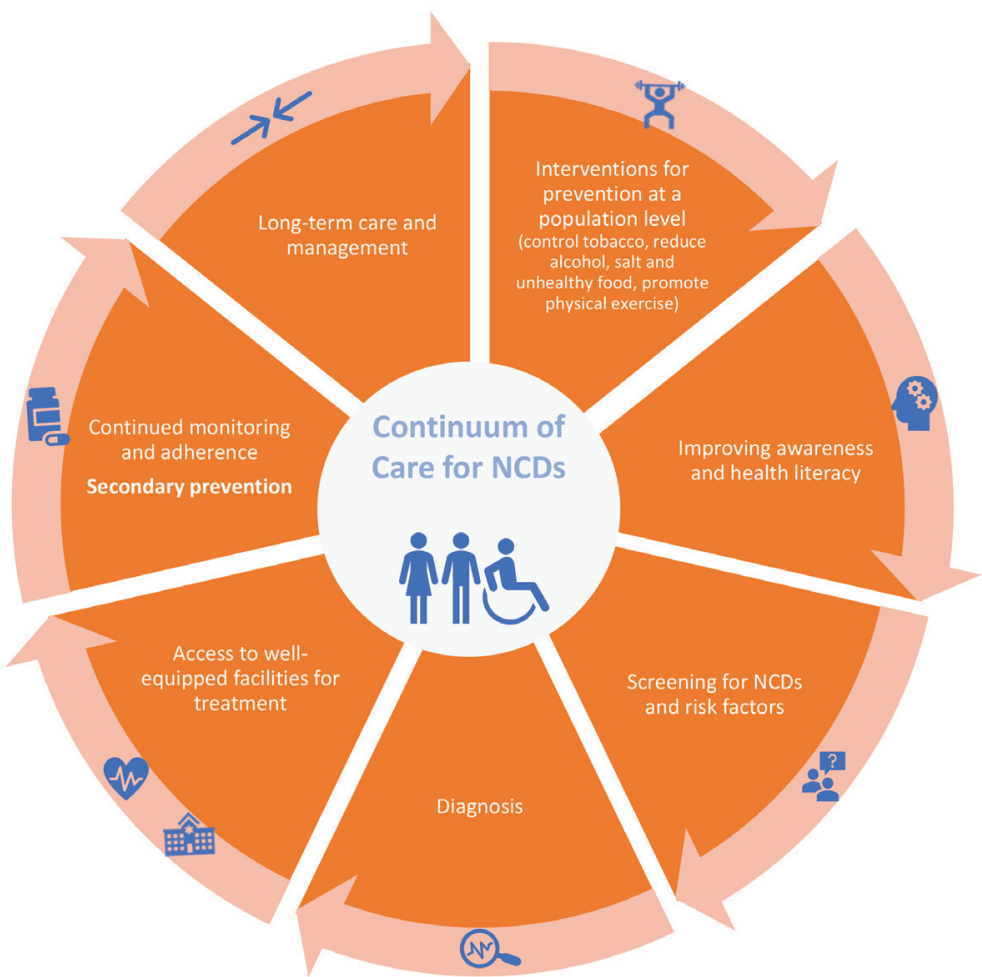

Figure 1. Continuum of care for the management of NCDs. Adapted from [4] with permission.

long-term follow up care are not part of the regular health system. Similarly, poverty and other social determinants of health is contributing to poor access to diagnosis and treatment of chronic conditions in Sub-Saharan Africa [3].

Implementation of NCD prevention policies and adoption of the WHO "best buy" interventions varies by country within, and across, the region. There are still gaps in terms of implementation of the framework convention for the tobacco control (FCTC) and policies to curb the harmful use of alcohol, as reported in five African countries [8]. Implementation of policy interventions for both diet and physical activity have been less prioritized. In most instances, there are no population level data enabling evaluation of these population level interventions to strengthen the strategies [9].

Low-and middle-income countries (LMICs) face a major barrier in accessing diagnosis, treatment and care; in countries such as Bhutan and Nepal, more than $60 \%$ of adults with hypertension remain unaware of their condition [10]. Similarly, more than half of the cohort with hypertension in the HealthRise project in South Africa were unaware of their status [4]. Among those aware, a significant proportion never accessed treatment or were otherwise lost to follow-up [4]. Thus, there remains a wide gap in achieving treatment and control of hypertension.

Many countries have inadequate health services to support NCD management. In Tanzania, health facilities at all levels appear ill prepared to address the diagnosis, treatment, and ongoing monitoring needs of patients with NCDs [11]. Specific to hypertension, in

Transferring tasks to community health volunteers, who have primarily been active in controlling infectious diseases and improving maternal and child health, may potentially be effective in improving diagnosis, monitoring and adherence to treatment for NCDs in LMICs. a survey of health facilities in Nepal, not all facilities had digital or manual blood pressure devices [12]. Only about one in five health facilities offered services for the diagnosis and/ or management of diabetes with less than a quarter of those sites, having stock of essential anti-diabetic medicines [12]. The declining rate of mortality in HICs is attributed to their effective prevention strategies, prompt diagnosis, referral services and long-term care services that are lacking in many LMICs. Successful implementation of prevention strategies for NCDs lie within the political will of countries to curb alcohol, 


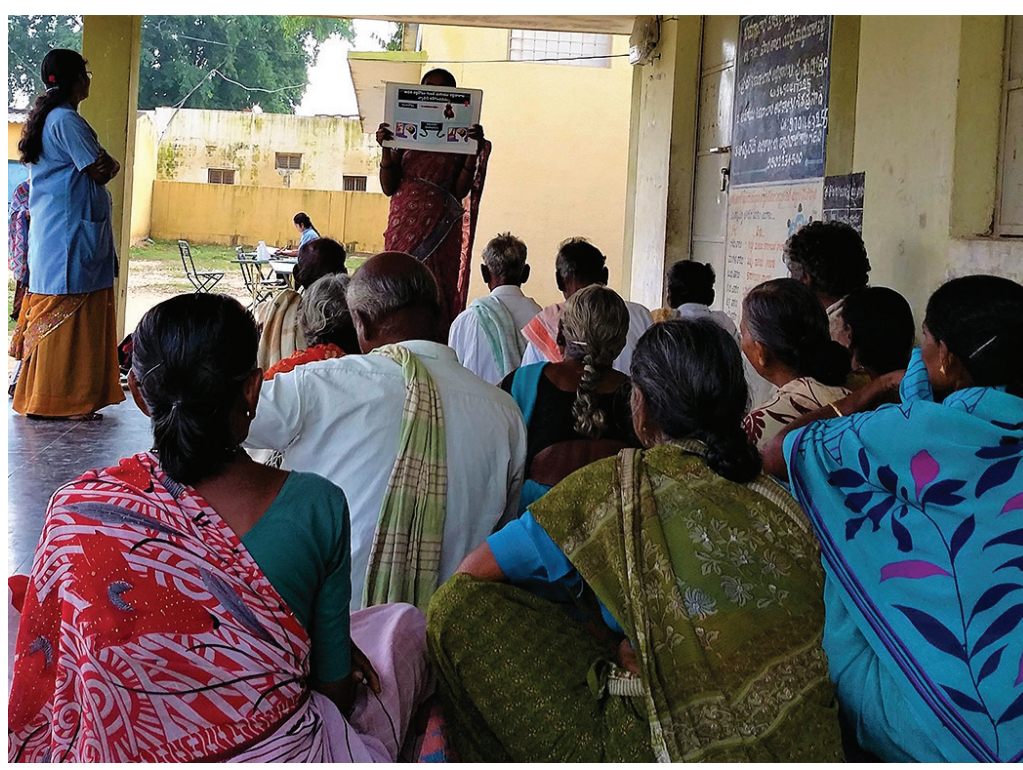

Photo: From the collection of Oduru Suresh, used with permission. tobacco use, discourage unhealthy foods and drinks, and promote physical activity. Equally important is strengthening the health system to respond to treatment needs and long-term monitoring to achieve control of risk factors. Improving adherence through patient education and communication, improving availability and affordability of medication and continuous monitoring and follow up is critical for the control of hypertension and other cardiovascular deaths [3]. Transferring tasks to community health volunteers, who have primarily been active in controlling infectious diseases and improving maternal and child health, may potentially be effective in improving diagnosis, monitoring and adherence to treatment for NCDs in LMICs [13].

Single fragmented approaches to achieve the SDG goals are not working. Strategies must be strengthened to improve outcomes across the NCD continuum, from prevention through to long-term care. This must include a robust monitoring system, with indicators set for each process in the pathway of care. Without this, we will not achieve the target to reduce NCD related mortality by one third by 2030.

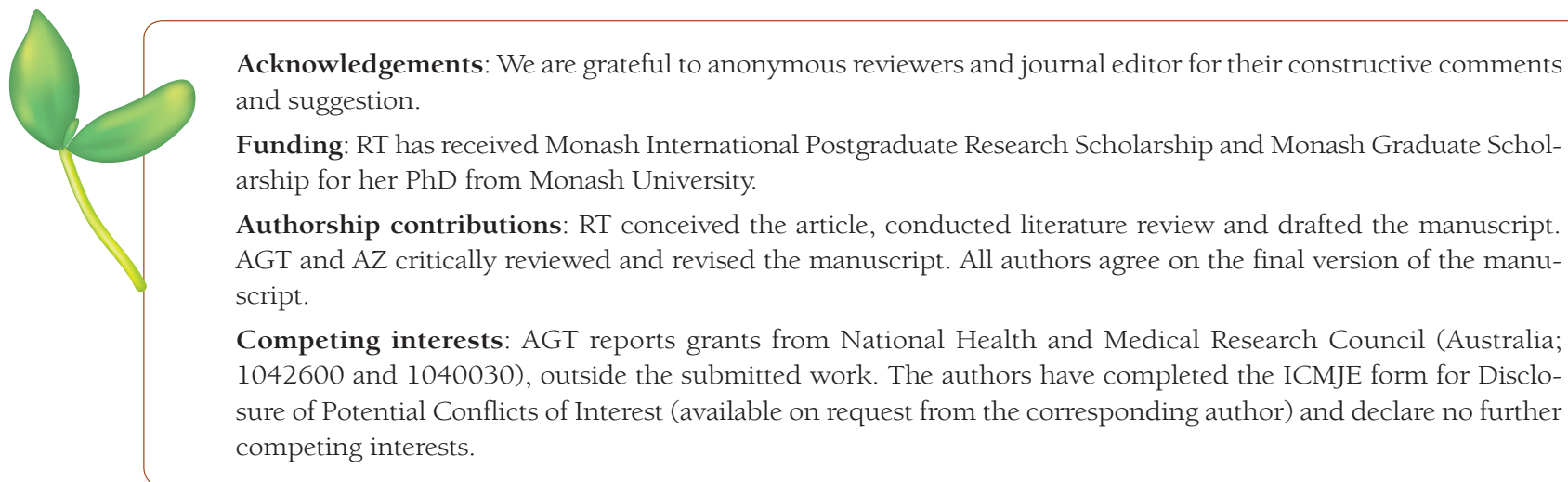

1 NCD Countdown 2030 collaborators. NCD Countdown 2030: worldwide trends in non-communicable disease mortality and progress towards Sustainable Development Goal target 3.4. Lancet. 2018;392:1072-88. Medline:30264707 doi:10.1016/S0140-6736(18)31992-5

2 Mensah GA, Wei GS, Sorlie PD, Fine LJ, Rosenberg Y, Kaufmann PG, et al. Decline in cardiovascular mortality: possible causes and implications. Circ Res. 2017;120:366-80. Medline:28104770 doi:10.1161/CIRCRESAHA.116.309115

3 Seedat Y, Ali A, Ferdinand KC. Hypertension and cardiovascular disease in the sub-Saharan African context. Ann Transl Med. 2018;6:297. Medline:30211185 doi:10.21037/atm.2018.06.45

4 Wollum A, Gabert R, McNellan CR, Daly JM, Reddy P, Bhatt P, et al. Identifying gaps in the continuum of care for cardiovascular disease and diabetes in two communities in South Africa: Baseline findings from the HealthRise project. PLoS One. 2018;13:e0192603. Medline:29538420 doi:10.1371/journal.pone.0192603

5 McNairy ML, El-Sadr WM. The HIV care continuum: no partial credit given. AIDS. 2012;26:1735-8. Medline:22614888 doi:10.1097/QAD.0b013e328355d67b

6 Ford ES, Ajani UA, Croft JB, Critchley JA, Labarthe DR, Kottke TE, et al. Explaining the decrease in US deaths from coronary disease, 1980-2000. N Engl J Med. 2007;356:2388-98. Medline:17554120 doi:10.1056/NEJMsa053935

7 Owolabi MO. Taming the burgeoning stroke epidemic in Africa: stroke quadrangle to the rescue. West Indian Med J. 2011;60:412-21. Medline:22097671

8 Juma PA, Mohamed SF, Mwagomba BLM, Ndinda C, Mapa-Tassou C, Oluwasanu M, et al. Non-communicable disease prevention policy process in five African countries. BMC Public Health. 2018;18:961. Medline:30168393 doi:10.1186/ s12889-018-5825-7 
un tion policies: World Health Organization; 2017.

10 World Health Organization. Tracking universal health coverage: first global monitoring report: Geneva: World Health Organization; 2015.

11 Peck R, Mghamba J, Vanobberghen F, Kavishe B, Rugarabamu V, Smeeth L, et al. Preparedness of Tanzanian health facilities for outpatient primary care of hypertension and diabetes: a cross-sectional survey. Lancet Glob Health. 2014;2:e28592. Medline:24818084 doi:10.1016/S2214-109X(14)70033-6

12 Ministry of Health, Nepal Health Sector Support Program (NHSSP), ICF. Nepal Health Facility Survey 2015. Kathmandu: Ministry of Health; 2017. Available: https://dhsprogram.com/pubs/pdf/SPA24/SPA24.pdf. Accessed: 20 November 2019.

13 Neupane D, McLachlan CS, Mishra SR, Olsen MH, Perry HB, Karki A, et al. Effectiveness of a lifestyle intervention led by female community health volunteers versus usual care in blood pressure reduction (COBIN): an open-label, clusterrandomised trial. Lancet Glob Health. 2018;6:e66-73. Medline:29241617 doi:10.1016/S2214-109X(17)30411-4

\section{Correspondence to:}

Rajshree Thapa

Department of Medicine

Monash University

27-31 Wright Street

Clayton, VIC 3168

Australia

rajshree.thapa@monash.edu 